\title{
ANALYTICAL ENERGY GRADIENTS FOR A UNITARY COUPLED-CLUSTER THEORY
}

\author{
Mark R. HOFFMANN and Jack SIMONS \\ Department of Chemistry, University of Utah, Salt Lake City, UT 84112, USA
}

Received 9 September 1987; in final form 16 October 1987

\begin{abstract}
Our recently developed and tested unitary multiconfigurational coupled-cluster electronic wavefunction method is extended to permit, for the first time, the analytical evaluation of energy derivatives. The unitary nature of this method admits a variational energy functional whose stationary nature plays a key role in simplifying our derivation. Explicit expressions are given for the gradient (first energy derivative) for both the full unitary coupled cluster and its coupled electron pair approximation (CEPA).
\end{abstract}

Recently, we [1] developed a theory of a unitary coupled-cluster (UCC) method and demonstrated its computational applicability via illustrative calculations on the well-studied [2-6] $\mathrm{BeH}_{2}$ and $\mathrm{H}_{2} \mathrm{O}$ species. The unitary nature of our method, and the resultant variational energy expression, distinguish our approach from those of most others and allow us to straightforwardly obtain a compact analytical expression for the UCC energy gradient.

The importance of analytic derivatives in investigating chemical phenomena on a Born-Oppenheimer potential-energy hypersurface is widely appreciated [7,8]. In this Letter, we demonstrate that a class of energy functionals, of which our UCC energy is a member, can be readily differentiated using procedures similar to those used in evaluating configuration interaction $(\mathrm{CI})[9,10]$ and multiconfiguration self-consistent field (MC SCF) [10-14] energy derivatives. We provide explicit formulas for the UCC gradient in terms of integrals and density matrices over molecular and atomic orbitals as well as orbital and configuration amplitude responses which are obtained through coupled perturbed MC SCF methods [11-14]. Energy-derivative expressions for an important approximation to the coupled-cluster wavefunction, the coupled electron pair approximation (CEPA) [15], are also included.

The variational condition that the unitary coupled-cluster energy (ref. [1], eq. (2.11)) is stationary with respect to variations in the cluster amplitudes $\left(\partial E_{\mathrm{CC}} / \partial t_{K}=0\right)$ was shown in ref. [1] to lead to the following expression for the UCC energy:

$$
E_{\mathrm{CC}}=\left\langle\Phi_{\mathrm{MC}}|\exp (-T) H \exp (T)| \Phi_{\mathrm{MC}}\right\rangle=E_{\mathrm{MC}}+\sum_{K}\left\langle\Phi_{\mathrm{MC}}|H| K\right\rangle \tau_{K},
$$

which is valid through second order in the $\tau_{K}$ amplitudes. Here the summation is over the configuration state functions (CSFs) in the space which interacts with the MC SCF reference function $\left|\Phi_{M C}\right\rangle$ through the Hamiltonian $H$, and $\tau_{K}$ is an amplitude of our UCC cluster operator $T$ coupling $\left|\Phi_{\mathrm{MC}}\right\rangle$ to $|K\rangle: \tau_{K}=\left\langle K|T| \Phi_{\mathrm{MC}}\right\rangle$. In eq. (1), $E_{\mathrm{MC}}$ is the energy of the MC SCF reference state.

In the CEPA version [16] of our UCC method [1], the $\tau_{K}$ are calculated directly as variational parameters; in the UCC method, the $\tau_{K}$ are obtained as $\left\langle K|T| \Phi_{\mathrm{MC}}\right\rangle$ after the variational parameters $t_{\alpha}$ (which define $T=\Sigma_{\alpha} t_{\alpha} e_{\alpha}$ in terms of generators $e_{i j}$ and single products of such generators of the unitary group) are computed by solving the linear equations of ref. [1]. Both the CEPA and UCC methods give identical energy expressions.

The conventional one- and two-electron operators forming the Hamiltonian may be introduced to give

$$
E_{\mathrm{CC}}=E_{\mathrm{MC}}+\sum_{i j} h_{i j} D_{i j}+\sum_{i j k l}(i j \mid k l) d_{i j k l},
$$


a form reminiscent of the CI or MC SCF energy expressions, although here the density matrices $d_{i j k l}$ and $D_{i j}$ are of the transition density matrix form. Explicitly

$$
D_{i j}=\sum_{K} \tau_{K} \sum_{l} C_{I}\left\langle I\left|e_{i j}\right| K\right\rangle,
$$

and

$$
d_{i j k l}=\frac{1}{4} \sum_{K} \tau_{K} \sum_{I} C_{l}\left(\left\langle I\left|e_{i j k l}\right| K\right\rangle+\left\langle I\left|e_{i j k l}\right| K\right\rangle\right),
$$

where $C_{l}$ is the CSF amplitude in the MC SCF reference function and $e_{i j}$ and $e_{i j k l}$ are generators and generator products of the unitary group. The geometry dependence of $E_{\mathrm{CC}}$ is contained in the $\tau_{K}$, the $C_{l}$ and the integrals $h_{i j}$ and $(i j \mid k l)$.

Application of the chain rule for differentiation and using the fact that the energy has been made stationary with respect to variations in the $\tau_{K}$ or $t_{\alpha}$ cluster amplitudes $\left(\partial E_{\mathrm{CEPA}} / \partial \tau_{K}=0\right.$ or $\left.\partial E_{\mathrm{CC}} / \partial t_{\alpha}=0\right)$ allows one to write

$$
\frac{\mathrm{d} E_{\mathrm{CC}}}{\mathrm{d} a}=\frac{\mathrm{d} E_{\mathrm{MC}}}{\mathrm{d} a}+\sum_{i j} h_{i j}^{v_{a}} D_{i j}+\sum_{\mu \nu} h_{\mu \nu}^{a} D_{\mu \nu}+\sum_{i j} h_{i j} \frac{\partial D_{i j}}{\partial a}+\sum_{i j k l}(i j \mid k l)^{v_{a}} d_{i j k l}+\sum_{\mu \nu} d_{\mu \nu \rho \sigma}(\mu \nu \mid \rho \sigma)^{a}+\sum_{i j k l}(i j \mid k l) \frac{\partial d_{i j k l}}{\partial a} .
$$

The variational nature of $E_{\mathrm{CC}}$ with respect to the $\tau_{K}$ (or $t_{\alpha}$ ) amplitudes obviates the need to solve for the response of the CC amplitudes with respect to geometrical displacement. Only the orbital responses $U_{i j}^{a}$ and the MC SCF configuration amplitude responses $\partial C_{l} / \partial a$ need to be obtained [11,12]. The practical evaluation of our unitary CC energy derivative (eq. (4)) requires little more than the effort required for the MC SCF gradient and $U_{i j}^{a}$ and $\partial C_{l} / \partial a$ evaluation. This is a very important practical feature of our result.

In eq. (4), the $h_{i j}^{U_{a}}$ and $(i j \mid k l)^{v_{a}}$ are partially transformed $[10,13,14]$ one- and two-electron integrals in which the orbital responses $\sum_{j} U_{j i}^{a} \phi_{j}$ have been included as described in ref. [13], $D_{\mu \nu}$ and $d_{\mu \nu \rho \sigma}$ are density matrices which have been back-transformed to the atomic orbital basis [9]. For unitary CEPA energies, for which the $\tau_{K}$ themselves are the independent variational parameters, the transition density matrix derivatives $\partial D_{i j} / \partial a$ and $\partial d_{i k l} / \partial a$ are expressed as in eq. (3) but with $\partial C_{I} / \partial a$ in place of $C_{I}$. Since the $\tau_{K}$ in a UCC calculation are not the independent variational parameters (the $t_{\alpha}$ are), the derivative density matrices contain both this $\partial C_{I} / \partial a$ factor plus a second term of the same form as in eq. (3) but with $\partial \tau_{K} / \partial a=\Sigma_{L}\langle K|T| L\rangle \partial C_{L} / \partial a$ in place of $\tau_{K}$. With these specifications, eq. (4) gives or working result for the UCC and variational CEPA first energy derivative.

We again stress that all quantities in eq. (4) are available either from the MC SCF gradient calculation, the UCC energy calculation, or by performing the coupled perturbed multiconfiguration Hartree-Fock (CP MC HF) response calculation [10-12] to compute the molecular orbital and $\mathrm{CI}$ response quantities $\left(\partial C_{I} / \partial a\right.$ and $U_{i j}^{a}$ ). The usual form of the CP MC HF equations [10-13] (i.e. as used in MC SCF second derivatives and in $\mathrm{CI}$ first derivatives from an $\mathrm{MC}$ reference) may be used straightforwardly whenever the electronic energy expression is invariant to unitary transformations within the core valence, and virtual molecular orbital subsets. This is, in fact, the case in the CEPA implementation of our UCC method.

The implementation of our UCC method as expressed in ref. [1] may, for practical reasons, utilize a (numerically) screened manifold of cluster operators. As a result, the UCC energy is, in general, not invariant to arbitrary orbital rotations because this screening process will usually destroy invariance within orbital subsets. Therefore, a specific choice of the orbitals (e.g., some physically motivated choice of canonical orbitals) must be made and preserved at all nuclear geometries in order to have a consistently defined wavefunction. As described in our earlier paper, the specific choice of orbitals that we make diagonalizes the core Fock matrix $[11,17]$,

$$
F_{i j}^{c}=h_{i j}+\frac{1}{2} \sum_{k l}^{o c c} \lambda_{k l}[2(i j \mid k l)-(i k \mid j l)] \text {, }
$$


within both the core and virtual orbital spaces, and diagonalizes the Lagrangian matrix $[11,18]$,

$$
\epsilon_{i j}=\sum_{k} h_{i k} \gamma_{k j}+2 \sum_{k l m}(i k \mid l m) \Gamma_{j k l m},
$$

in the valence orbital space.

The condition that the molecular orbitals at the (infinitesimally) displaced geometries also diagonalize $F_{i j}^{c}$ and $\epsilon_{i j}$ and hence that the coupled-cluster operator manifold at the slightly displaced geometry is consistent with the manifold at the original geometry, gives rise to conditions on the derivatives of the core Fock matrix [11],

$$
\mathrm{d} F F_{i j}^{\mathrm{c}} / \mathrm{d} a=0, \quad i \neq j
$$

and on the derivatives of the sum of the off-diagonal Lagrangian matrix,

$$
\mathrm{d}\left(\epsilon_{i j}+\epsilon_{j i}\right) / \mathrm{d} a=0, \quad i \neq j .
$$

The difference of the off-diagonal Lagrangian matrix elements must also vanish (at all powers of differentiation), a condition already used in defining the CP MC HF equations [11].

Differentiating the core Fock matrix, as indicated in eq. (7), gives

$$
\begin{aligned}
0 & =\sum_{\mu \nu} C_{\mu}^{i} D_{\nu}^{j}\left(h_{\mu \nu}^{a}+\sum_{\rho \sigma} \gamma_{\rho \sigma}\left[(\mu \nu \mid \rho \sigma)^{a}-\frac{1}{2}(\mu \rho \mid \nu \sigma)^{a}\right]\right)+\sum_{k l}\left(\sum_{l j} \frac{\partial C_{l}}{\partial a} C_{J}\left(\gamma_{k l}^{I J}+\gamma_{k l}^{J l}\right)\right)\left[(i j \mid k l)-\frac{1}{2}(i k \mid j l)\right] \\
& +\sum_{s} U_{s i}^{a}\left(\sum_{k l} \gamma_{k l}\left[(s j \mid k l)-\frac{1}{2}(s k \mid j l)\right]+h_{s j}\right)+\sum_{s} U_{s j}^{a}\left(\sum_{k l} \gamma_{k l}\left[(i s \mid k l)-\frac{1}{2}(i k \mid s l)\right]+h_{i s}\right) \\
& +\sum_{s r} U_{s r}^{a}\left(\sum_{T} \gamma_{r l}\left[2(i j \mid s l)-\frac{1}{2}(i s \mid j l)-\frac{1}{2}(i l \mid j s)\right]\right)
\end{aligned}
$$

for all $i>j, i, j \in$ core and $i, j \in$ virtual. In eq. (9), the Greek indices run over all atomic orbitals, $k, l, r$ denote molecular orbitals occupied in some or all CSFs, and $s$ runs over all molecular orbitals. The terms in eq. (9) are identical to those derived earlier by Osamura and co-workers [11] in the context of derivatives of a $\mathrm{CI}$ wavefunction using MC SCF orbitals. However, in the present case, the separation into energy redundant and non-redundant variables does not occur. Thus, eq. (9) represents a generalization of the coupled perturbed multiconfigurational Hartree-Fock (CP MC HF) equations of Osamura and co-workers for the $U_{i j}^{a}$ and $\partial C_{l} / \partial a$.

Similarly, the sum of the off-diagonal Lagrangian matrix elements can be differentiated to give

$$
0=\sum_{s} U_{s i}^{a} \epsilon_{s j}+\sum_{s r} U_{s r}^{a} Y_{i j s r}+\epsilon_{i j}^{a}+\sum_{l} \frac{\partial C_{l}}{\partial a} Y_{i j}^{l}+(i \rightleftarrows j),
$$

for all $i>j, i, j \in$ valence. In eq. (10), we use the same index convention described in the preceding paragraph.

Examination of eqs. (9) and (10) shows that the usual energy non-redundant CP MC HF equations must be modified by adding $n_{\text {core }}\left(n_{\text {core }}+1\right) / 2+n_{\text {valence }}\left(n_{\text {valence }}+1\right) / 2+n_{\text {virtual }}\left(n_{\text {virtual }}+1\right) / 2$ equations to preserve the canonical nature of the orbitals. Further examination of eqs. (9) and (10), with consideration of the orbital rotation orthonormality condition, $U_{i j}^{a}+U_{j i}^{a}+S_{i j}^{a}=0$ (where $S_{i j}^{a}$ is the usual derivative overlap matrix transformed into the MO basis), shows that the number of orbital rotations $\left(U_{i j}^{a}\right)$ introduced in excess of the energy non-redundant ones exactly equals the number of additional equations. Thus it is to be expected that the larger set of simultaneous equations has a unique well-defined solution. Parenthetically we note that the quantities used to construct the coefficients in the additional equations and the modifications to existing CP MC HF equations are already available within conventional computer codes for the construction of the usual CP MC HF equations $[11,12]$. The solutions to these modified equations are then the orbital $\left(U_{i j}^{a}\right)$, and configuration amplitude $\left(\partial C_{I} / \partial a\right)$ changes which may then be used directly in computing the derivative energy, as in eq. (4). 
In summary, we have demonstrated in this Letter that the UCC molecular gradient, or its variational CEPA counterpart, are indeed practical within the framework of our UCC method. The unitary method and its resulting variational energy functional are the key ingredients to obtaining a computationally practical energy gradient expression. Computational implementation of this method is eminently viable due to both the straightforward functional form of the energy gradient expression and to the availability of key quantities from extant MC SCF analytical derivative codes.

We acknowledge the financial support of the National Science Foundation (CHE-8511307) and the US Army Research Office (DAAG-2984K0086), and the donors of the Petroleum Research Office Grant (PRF 14446AC6) administered by the American Chemical Society.

\section{References}

[1] M.R. Hoffmann and J. Simons, A Unitary Multiconfiguration Coupled Cluster Method: Theory and Applications, J. Chem. Phys., to be published.

[2] G.D. Purvis and R.J. Bartlett, J.Chem. Phys. 76 (1982) 1910; G.D. Purvis, R. Shepard, F.B. Brown and R.J. Bartlett, Intern. J. Quantum Chem. 23 (1983) 835;

Y.S. Lee and R.J. Bartlett, Intern. J. Quantum Chem. Symp. 17 (1983) 347.

[3] A. Banerjee and J. Simons, Chem. Phys. 81 (1983) 297; 87 (1984) 215.

[4] M.R. Hoffmann, X.-C. Wang and K.F. Freed, Chem. Phys. Letters 136 (1987) 392.

[5] P. Saxe, H.F. Schaefer III and N.C. Handy, Chem. Phys. Letters 79 (1981) 202; R.J. Harrison and N.C. Handy, Chem. Phys. Letters 95 (1983) 386.

[6] R.J. Bartlett, H. Sekino and G.D. Purvis, Chem. Phys. Letters 98 (1983) 66; W.D. Laidig and R.J. Bartlett, Chem. Phys. Letters 104 (1984) 424.

[7] H.F. Schaefer III, Quantum chemistry (Clarendon Press, Oxford, 1984).

[8] P. Jørgensen and J. Simons, eds., Geometrical derivatives of energy surfaces and molecular properties (Reidel, Dordrecht, 1986).

[9] B.R. Brooks, W.D. Laidig, P. Saxe, N.C. Handy and H.F. Schaefer III, Physica Scripta 21 (1980) 312;

B.R. Brooks, W.D. Laidig, P. Saxe, J.D. Goddard, Y. Yamaguchi and H.F. Schaefer III, J. Chem. Phys. 72 (1980) 4652.

[10] P. Jergensen and J. Simons, J. Chem. Phys. 79 (1983) 334.

[11] Y. Osamura, Y. Yamaguchi and H.F. Schaefer III, J. Chem. Phys. 77 (1982) 383.

[12] M.R. Hoffmann, D.J. Fox, J.F. Gaw, Y. Osamura, Y. Yamaguchi, R.S. Grev, G. Fitzgerald, H.F. Schaefer III, P.J. Knowles and N.C. Handy, J. Chem. Phys. 80 (1984) 2660.

[13] M. Page, P. Saxe, G.F. Adams and B.H. Lengsfield, J. Chem. Phys. 81 (1984) 434.

[14] T.U. Helgaker and J. Almlöf, Intern. J. Quantum Chem. 26 (1984) 275.

[15] W. Kutzelnigg, in: Methods of electronic structure theory, Vol. 3, ed. H.F. Schaefer III (Plenum Press, New York, 1977) pp. 129-188, and references therein.

[16] M.R. Hoffmann and J. Simons, to be submitted for publication.

[17] P. Siegbahn, A. Heilberg, B. Roos and B. Levy, Physica Scripta 21 (1980) 323.

[18] K. Reudenberg, L.M. Cheung and S.T. Elbert, Intern. J. Quantum Chem. 16 (1979) 1069. 